\title{
BMJ Open Piloting gender-oriented colorectal cancer screening with a faecal immunochemical test: population-based registry study from Finland
}

\author{
Tytti Sarkeala (D) , ${ }^{1}$ Martti Färkkilä, ${ }^{2}$ Ahti Anttila, ${ }^{3}$ Marja Hyöty, ${ }^{4}$ Matti Kairaluoma, ${ }^{5}$ \\ Tero Rautio, ${ }^{6}$ Markku Voutilainen, ${ }^{7}$ Sanni Helander, ${ }^{3}$ Maija Jäntti (D) ${ }^{3}$ \\ Milla Lehtinen, ${ }^{3}$ Lotta Patrikka, ${ }^{3}$ Nea Malila, ${ }^{3}$ Sirpa Heinävaara ${ }^{3}$
}

To cite: Sarkeala T, Färkkilä M, Anttila A, et al. Piloting gender-oriented colorectal cancer screening with a faecal immunochemical test: population-based registry study from Finland. BMJ Open 2021;11:e046667. doi:10.1136/ bmjopen-2020-046667

- Prepublication history for this paper is available online. To view these files, please visit the journal online (http://dx.doi. org/10.1136/bmjopen-2020046667).

Received 05 November 2020 Revised 25 December 2020 Accepted 20 January 2021
Check for updates

(C) Author(s) (or their employer(s)) 2021. Re-use permitted under CC BY-NC. No commercial re-use. See rights and permissions. Published by BMJ.

For numbered affiliations see end of article.

Correspondence to Dr Tytti Sarkeala;

tytti.sarkeala@cancer.fi

\section{ABSTRACT}

Objective To assess the feasibility and evaluate the performance of a relaunched colorectal cancer (CRC) screening programme with different cut-offs for men and women.

Design Population-based registry study.

Setting Nine municipalities in Finland which started CRC screening with faecal immunochemical test (FIT) in April 2019 with cut-off levels $70 \mu \mathrm{g} \mathrm{Hg} / \mathrm{g}$ faeces for men and $25 \mu \mathrm{g} \mathrm{Hg} / \mathrm{g}$ faeces for women.

Participants Men ( $n=13059)$ and women $(n=14669)$ aged 60-66 years invited to screening during the first programme year.

Outcome measures Participation rates, positivity rates, detection rates of CRC and advanced adenoma (AA), and positive predictive values (PPV) of FIT for CRC and AA.

Results Altogether 21993 invitees returned stool samples. The participation rate of women $(83.4 \%$; $95 \%$ Cl 82.8 to 84.0) was significantly higher than that of men (74.7\%; $95 \% \mathrm{Cl} 73.9$ to 75.4$)$. The positivity rates were $2.4 \%$ (2.2 to 2.7) and 2.8\% (2.5 to 3.1), respectively. In total, 37 CRCs and 116 AAs were detected. The detection rates of CRC and AA per 1000 participants were 1.8 (1.1 to 2.9) and 7.2 (5.6 to 9.1) for men and 1.6 (0.9 to 2.4) and 3.8 (2.8 to 5.0) for women. The PPVs per 100 positive tests were 6.6 (4.0 to 10.3) and 25.7 (20.6 to 31.4) for men and 6.4 (3.9 to 9.8) and 15.5 (11.6 to 20.2) for women.

Conclusions The chosen FIT strategy narrowed the gap in the diagnostic performance between men and women especially in the detection of CRC. The participation rates were excellent. The levels of positivity and detection rates were moderate and need further action. The results indicate that gender-specific protocols can be introduced to organised CRC screening. It is yet to be seen whether they are more effective than a uniform screening protocol.

\section{INTRODUCTION}

Since 2003, the European Union has recommended colorectal cancer (CRC) screening to its member states as a public health policy. ${ }^{1}$ There are several methods available for CRC screening. Stool-based tests to detect blood include the guaiac faecal occult blood test
Strengths and limitations of this study

- Men have been shown to benefit more from faecal immunochemical test (FIT) screening than women; we used gender-specific protocols to narrow this outcome gap.

- Our study is the first to report performance estimates of FIT screening based on different cut-offs for men and women.

- We were able to link registry-based individual screening data with individual cancer and population registry data to verify cancer diagnoses and deaths.

- Piloting enables us to create a feasible screening strategy for both genders before implementation of the national programme.

- We assume that the participants of the nine piloting municipalities represent the rest of Finland even though there was a small discrepancy in the risk profiles of colorectal cancer between these two population groups.

(gFOBT) and faecal immunochemical test (FIT). In recent years, FIT has often replaced gFOBT since it has shown increased sensitivity for detection of adenomas and higher acceptance and higher yield of colorectal neoplasms. ${ }^{2}$ Further advantages over gFOBT include a non-fixed haemoglobin concentration cut-off and the specificity to detect human haemoglobin. ${ }^{3}$

In Finland, biennial gFOBT screening was started as a randomised public health programme for the population aged 60-69 years in 2004. The reported performance estimates were comparable with those of other European screening programmes. ${ }^{4}$ The interim results on effectiveness in 2015 showed, however, similar CRC mortality in the screening and control arms and a nonsignificant increase in CRC mortality in women. Additionally, variation in the relative 
CRC mortality between the study arms could be attributed to gender $(\mathrm{p}=0.06) .{ }^{5}$ Due to these non-optimal results, the Finnish gFOBT programme was suspended in 2016.

Similar variation in the performance and outcome of CRC screening has also been reported elsewhere. European FIT studies have found higher sensitivity for advanced neoplasia and higher positive predictive value (PPV) in men compared with women. ${ }^{67}$ The gFOBT trial in the USA showed significantly different reduction in CRC mortality between men and women (37\% vs $8 \%$ ) after 30 years of follow-up. ${ }^{8}$ There are several potential reasons behind this discrepancy. For example, a greater proportion of adenomas are found in the right colon in women. ${ }^{9}$ Also sessile serrated lesions may be more prevalent in women. ${ }^{10}$ Therefore, adenomas in women may give less likely blood in stool and be less easy to detect during colonoscopy. Nonetheless, CRC screening programmes in Europe generally use the same approach for men and women. The restarted, FIT-based CRC screening programme in Finland aims to implement effective screening strategy for both genders. This study reports the design and the first-year results of the programme.

\section{MATERIALS AND METHODS}

Individually randomised (1:1) gFOBT CRC screening was launched in Finland in 2004. ${ }^{11}$ In the gradually expanding programme, men and women aged 60-69 years were invited to screening biennially. The programme covered approximately $50 \%$ of the target population in 2014 . During 2004-2014, altogether 466000 men and women were individually randomised either to the screening or the control arm.

In 2015, the published discrepancy between men and women on the interim effectiveness started a discussion on the future of CRC screening in Finland and eventually led to a shutdown of the gFOBT-based screening programme. During the year 2016, a group of experts analysed the scientific evidence concerning all cancer screening programmes. In December 2016, a decision to relaunch CRC screening with an FIT test was made in the Ministry of Social Affairs and Health.

\section{Planning phase}

In Spring 2017, a clinical and epidemiological expert group was established in the Finnish Cancer Registry. Municipalities attending the previous, randomised screening programme were contacted and asked to voluntarily join a pilot phase of FIT screening. Several laboratories were also contacted. Cost-benefit calculations based on existing CRC, screening and treatment information $^{12}$ (Mäklin et al 2019, manuscript) were performed for municipalities showing interest to participate. One screening laboratory, Fimlab Oy, was chosen to carry out the screening invitations and analyses in the pilot phase. Screening materials were planned and prepared in the Finnish Cancer Registry. ${ }^{13}$

The screening protocol was planned by the expert group in 2018. Based on the national incidence and life expectancy figures ${ }^{1415}$ and scientific evidence, ${ }^{16}$ the target population was extended to men and women aged 60-74 years. To assure availability of follow-up resources, they were to be invited to screening every 2 years with a gradually expanding scheme (implementation period of 9 years, 2019-2027; figure 1). A questionnaire concerning family history and symptoms of CRC as well as major lifestylerelated measures (alcohol use, smoking, body mass index) was included in the programme to measure associations between CRC risk and screening performance.

Gender-specific cut-offs were introduced to account for the plausible variation in carcinogenic pathways between men and women and the differences in CRC incidence levels (162 per 100000 in men, 110 per 100000 in women in 2017 at ages $60-74$ years) and to reach a similar relative reduction in CRC mortality for both genders. To find reasonable FIT cut-offs, all available information on agespecific cut-offs and test positivity rates in the existing European CRC screening programmes were addressed. ${ }^{17-21}$ Evidence from Nordic countries was of specific interest due to similar societal, lifestyle and healthcare structures and comparable incidence figures. ${ }^{22}$

Based on adequacy of colonoscopy resources and previous CRC screening results in Finland, the test positivity rates were set at $3 \%$ for men and $5 \%$ for women. The available information on FIT cut-off levels and positivity

\begin{tabular}{|l|c|c|c|c|c|c|c|c|c}
\hline & 2019 & 2020 & 2021 & 2022 & 2023 & 2024 & 2025 & 2026 & 2027 \\
\hline 1967 & 52 & 53 & 54 & 55 & 56 & 57 & 58 & 59 & 60 \\
\hline 1966 & 53 & 54 & 55 & 56 & 57 & 58 & 59 & 60 & 61 \\
\hline 1965 & 54 & 55 & 56 & 57 & 58 & 59 & 60 & 61 & 62 \\
\hline 1964 & 55 & 56 & 57 & 58 & 59 & 60 & 61 & 62 & 63 \\
\hline 1963 & 56 & 57 & 58 & 59 & 60 & 61 & 62 & 63 & 64 \\
\hline 1962 & 57 & 58 & 59 & 60 & 61 & 62 & 63 & 64 & 65 \\
\hline 1961 & 58 & 59 & 60 & 61 & 62 & 63 & 64 & 65 & 66 \\
\hline 1960 & 59 & 60 & 61 & 62 & 63 & 64 & 65 & 66 & 67 \\
\hline 1959 & 60 & 61 & 62 & 63 & 64 & 65 & 66 & 67 & 68 \\
\hline 1958 & 61 & 62 & 63 & 64 & 65 & 66 & 67 & 68 & 69 \\
\hline 1957 & 62 & 63 & 64 & 65 & 66 & 67 & 68 & 69 & 70 \\
\hline 1956 & 63 & 64 & 65 & 66 & 67 & 68 & 69 & 70 & 71 \\
\hline 1955 & 64 & 65 & 66 & 67 & 68 & 69 & 70 & 71 & 72 \\
\hline 1954 & 65 & 66 & 67 & 68 & 69 & 70 & 71 & 72 & 73 \\
\hline 1953 & 66 & 67 & 68 & 69 & 70 & 71 & 72 & 73 & 74 \\
\hline
\end{tabular}

Figure 1 Invitation matrix of the colorectal cancer screening programme by birth and calendar years. Shaded squares refer to those invited to screening. 

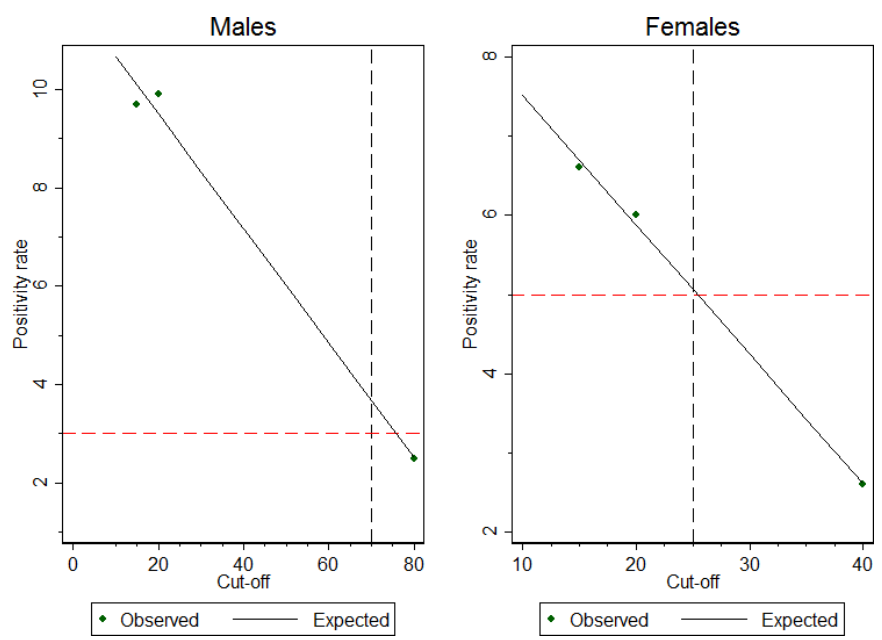

Figure 2 Graphic display of the prediction of cut-offs for men and women.

rates of men and women 60-69years old from Denmark, Norway and Sweden were used to predict cut-off levels for these rates for Finland ( $\varnothing$ Holme, 2018, personal communication). ${ }^{23}{ }^{24}$ Since the Swedish CRC incidence rates were closest to those in Finland, ${ }^{22}$ and Sweden had recently introduced different cut-off levels for men and women, ${ }^{24}$ the Swedish data were weighted twice as much in the calculations as data from other Nordic countries. The cut-off predictions were performed with weighted linear regression in Spring 2018 and they ended up to round numbers, cut-off-levels of $25 \mu \mathrm{g} \mathrm{Hg} / \mathrm{g}$ faeces for women and $70 \mu \mathrm{g} \mathrm{Hg} / \mathrm{g}$ faeces for men (figure 2). Thereafter, tendering of FIT test was performed by the screening laboratory, and FOB Gold (Sentinel Diagnostics, Milan, Italy) was chosen as a FIT test for the pilot phase.

\section{IT environment and follow-up protocol}

The IT (information technology) environment and division of tasks were planned together with the screening laboratory. According to these plans, the Finnish Cancer Registry provides information on screening invitees (personal identity code, name, address). The laboratory sends similar invitation packages to all men and women in the target population, performs analytics of stool samples and incorporates information on the whole screening chain (tests, referral examinations, and surgical or other further treatment) into the laboratory screening database. The database delivers these screening data regularly to the Finnish Cancer Registry for monitoring and evaluation (figure 3).

Each municipality has a contact nurse responsible for informing and advising the participants on positive test results and performing feasibility assessment on referral examinations. Based on previous protocols, exclusion criteria for further examinations include chronic inflammatory bowel disease, follow-up due to previous CRC and a colonoscopy examination performed within the last 3 years.

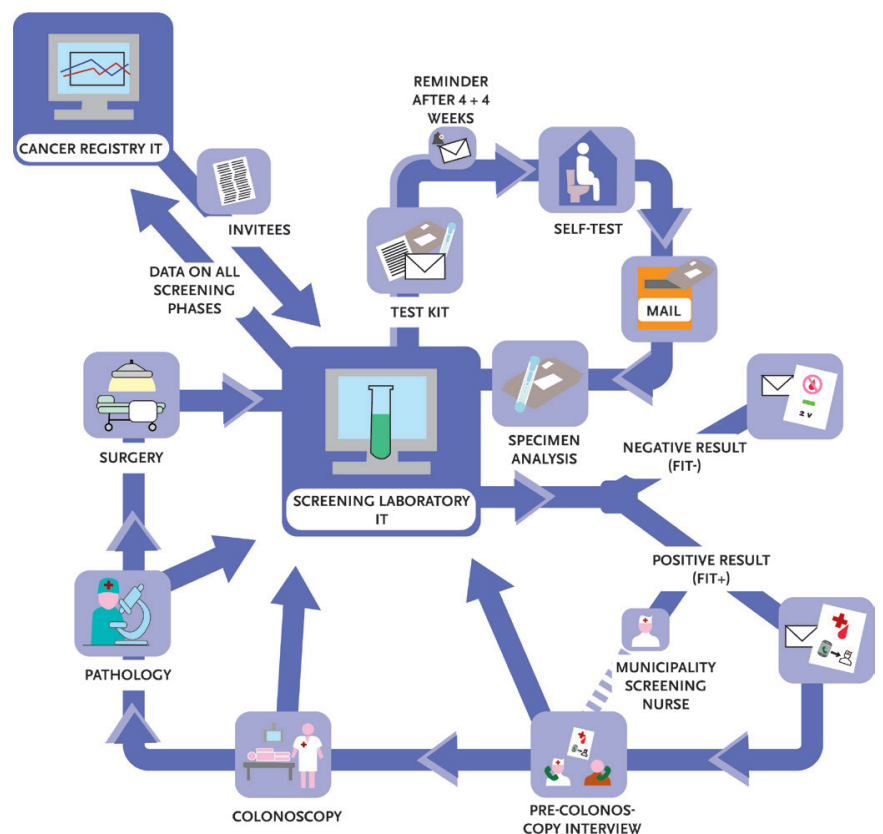

Figure 3 Graphic display of the IT workflow. FIT, faecal immunochemical test; IT, information technology.

Information on test results is mailed to the participants. The letter on positive test results includes a request to contact the nurse by phone. The referral examinations, mostly colonoscopies, are performed according to the protocol of each municipality. Histological samples obtained from colonoscopies are sent to the pathology unit. After analysis, the pathologist returns the diagnostic information to the endoscopists. The contact nurse enters the information into the laboratory database.

All colonoscopies are performed by accredited endoscopists. Training on tasks related to referral examinations is organised regularly for the endoscopists and the contact nurses.

Participants with CRC or larger adenomas needing endoscopic submucosal dissection procedure or partial colectomy are referred for further treatment and surveillance according to the guidelines of the European Society of Gastrointestinal Endoscopy.

\section{Data collection and analysis}

In April 2019, nine municipalities accepted the design and protocol of the CRC screening pilot and started screening. These municipalities were sparsely situated in West, East and Northern Finland and covered approximately 100000 Finnish men and women aged 60-74years. According to the gradual invitation scheme (figure 1), populations aged $60,62,64$ and 66 years were to be invited to screening during the first programme year.

The invitation package, including information on CRC screening, the FIT test kit, instructions to take the test, a return cardboard letter and a paper-print questionnaire or a password and instructions to fill in a web questionnaire, was posted to the invitees. The participants performed a single FIT test and filled in the paper-print or the web questionnaire at home and returned these 
thereafter to the laboratory. The first reminder letter was sent after 4 weeks and the second after further 4 weeks in case the test was not returned. If analysis of the test sample failed in the laboratory, at most two new invitation packages were resent to the participant.

Data on first-round screening invitees and participants born in 1953, 1955, 1957 and 1959 during the period from April 2019 to April 2020 were collected to assess participation to screening, positivity rate, participation to colonoscopy, detection rates of CRC and advanced adenoma (AA), and PPVs of the FIT test and colonoscopy for CRC and AA. The participation rate was defined as the number of men and women returning the stool sample divided by the number of men and women invited. All persons who returned their stool samples by the end of April 2020 were counted as participants. The positivity rate was defined as the number of participating men and women with a test result at or above the cut-off level divided by the number of participating men and women with an assessable stool sample. The participation rate for colonoscopy was formulated as the proportion of men and women who underwent colonoscopy out of those having a positive FIT.

AA was defined as any adenoma with histology showing $25 \%$ or greater villous component or high-grade dysplasia or adenoma with size of $10 \mathrm{~mm}$ or larger. Due to potential risk of development from premalignant to malignant state, ${ }^{25}$ and a relatively small number of cases, we also classified serrated lesions with dysplasia as AAs. Information on CRCs and AAs was gathered from histological diagnoses, either based on biopsy from colonoscopy or from surgery.

The detection rates of CRC or AA were defined as the proportion of men and women with CRC or AA out of those with an assessable stool sample. The PPV of FIT test for CRC or AA was calculated as the proportion of men and women with CRC or AA out of those with a positive test result. The PPV of colonoscopy for CRC or AA was calculated as the proportion of men and women with CRC or AA out of those who underwent colonoscopy.

Participation by reminder round, gender and age with 95\% binomial CIs was determined by descriptive analyses. Other outcomes were analysed accordingly by gender. All analyses were conducted using Stata V.14 statistical software.

\section{Patient and public involvement}

Patients and the public were not involved in the study.

\section{RESULTS}

\section{Flow of target population through the screening process}

During the first pilot year in April 2019-April 2020, the target population of the CRC pilot included altogether 27728 persons: 13059 men and 14669 women. Figure 4 shows the flow through the screening process.

Altogether $99.9 \%$ of participants had an assessable FIT. Of these, $568(2.6 \%)$ were tested positive. Of all persons

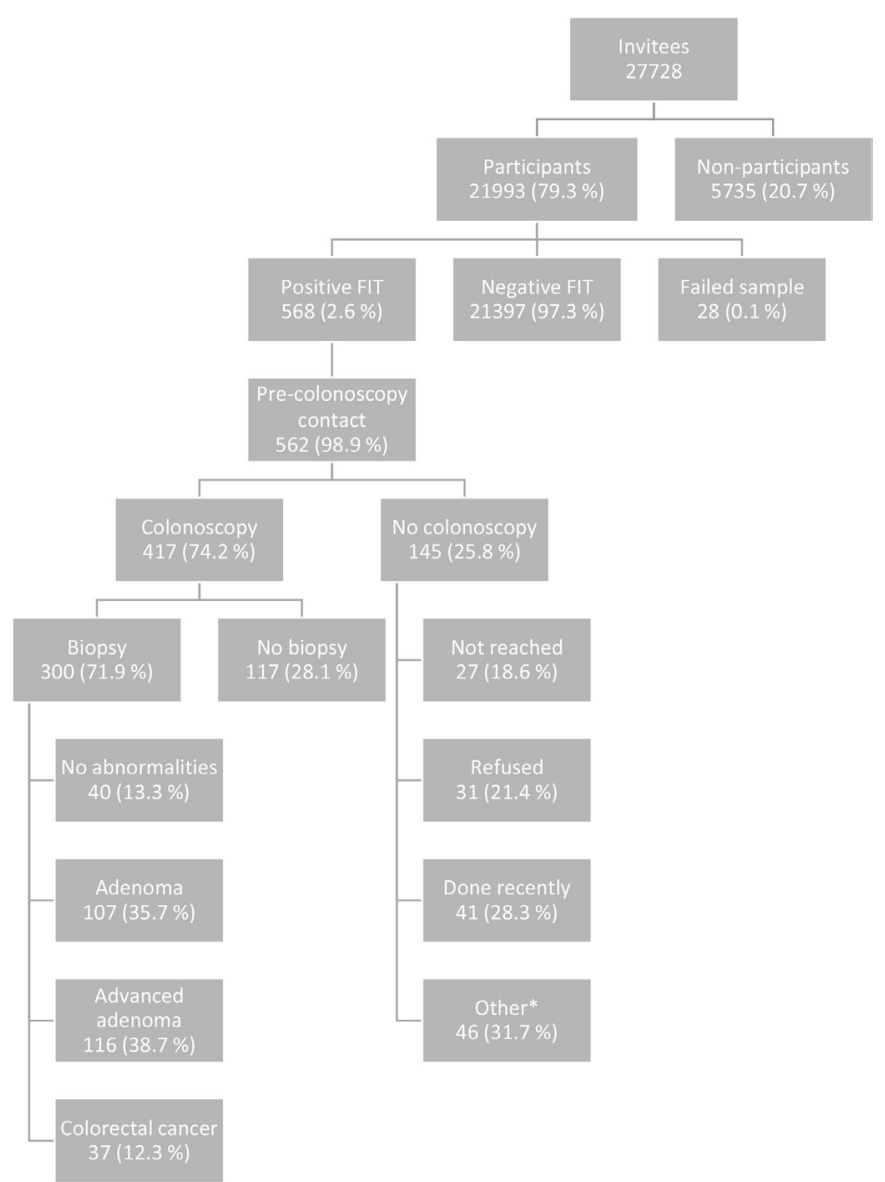

Figure 4 Flow chart of target population passing through the colorectal cancer screening chain. *Broken chain due to change of residence or healthcare unit, suspension of colonoscopy, or existing referral to colonoscopy outside the organised programme; active surveillance due to inflammatory bowel disease; poor health condition; and previously performed proctocolectomy. FIT, faecal immunochemical test.

receiving a positive result, $562(98.9 \%)$ had a precolonoscopy contact with a nurse. Of these, 417 persons $(74.2 \%)$ were further referred to colonoscopy. Excluding those for whom colonoscopy was not recommended or was taken elsewhere ( $n=87$, boxes 'other' and 'done recently' in figure 4), the overall uptake of colonoscopy was $87.8 \%$.

Of persons undergoing colonoscopies, $37.6 \%(n=157$, boxes 'no biopsy' and 'no abnormalities' in figure 4) were considered healthy, that is, having no abnormalities in the colon.

The median time between the date of analysis and the date of mailing of the positive test result was 14 days (data not shown). Accordingly, the median time between the date of mailing of the positive test result and the date of colonoscopy examination was 39 days (data not shown).

\section{Participation in screening}

A total of 21993 persons (79.3\%; 95\% CI 78.8 to 79.8) returned the stool sample to the screening laboratory (table 1$)$. The participation rate of women $(83.4 \%$; $95 \%$ 
Table 1 Participation ( $\mathrm{n}$ and \% with $95 \% \mathrm{Cl}$ ) in screening by gender, reminder round and age

\begin{tabular}{|c|c|c|c|c|c|c|}
\hline & \multicolumn{2}{|l|}{ Men } & \multicolumn{2}{|l|}{ Women } & \multicolumn{2}{|l|}{ All } \\
\hline & $\mathbf{n}$ & $\%(95 \% \mathrm{Cl})$ & $\mathbf{n}$ & $\%(95 \% \mathrm{Cl})$ & $\mathbf{n}$ & $\%(95 \% \mathrm{Cl})$ \\
\hline Total & 9754 & 74.7 (73.9 to 75.4$)$ & 12239 & 83.4 (82.8 to 84.0$)$ & 21993 & 79.3 (78.8 to 79.8$)$ \\
\hline With first reminder & 2534 & 19.4 (18.7 to 20.1$)$ & 2944 & 20.1 (19.4 to 20.7 ) & 5478 & 19.8 (19.3 to 20.2) \\
\hline $\begin{array}{l}\text { With second } \\
\text { reminder }\end{array}$ & 757 & 5.8 (5.4 to 6.2$)$ & 678 & 4.6 (4.3 to 5.0$)$ & 1435 & $5.2(4.9$ to 5.4$)$ \\
\hline 62 & 2452 & 74.6 (73.1 to 76.1$)$ & 3037 & 83.4 (82.1 to 84.6$)$ & 5489 & 79.2 (78.3 to 80.2$)$ \\
\hline 64 & 2501 & 77.5 (76.0 to 78.9$)$ & 3118 & $84.4(83.1$ to 85.5$)$ & 5620 & $81.2(80.2$ to 82.1$)$ \\
\hline 66 & 2401 & 76.2 (74.7 to 77.7$)$ & 3040 & 83.8 (82.6 to 85.0) & 5441 & 80.3 (79.3 to 81.2$)$ \\
\hline
\end{tabular}

CI 82.8 to 84.0$)$ was significantly higher than that of men (74.7\%; $95 \%$ CI 73.9 to 75.4$)$.

The use of reminders seemed to improve participation of both genders (table 1 ). The overall participation without reminders was $54.4 \%$, and after one reminder was $74.1 \%$. The second reminder increased the participation further by $5.2 \%$. By reminder round, participation increased by $25.2 \%$ (24.5 to 26.0 ) in men and $24.7 \%$ (24.0 to 25.4 ) in women.

There was no clear increase in the participation rate by age (table 1). The youngest age group of 60 years had, however, the lowest participation in both men and women.

\section{Positivity rates, PPVs and detection rates}

The overall positivity rate was higher in men $(2.8 \% ; 95 \%$ CI 2.5 to 3.1$)$ than in women $(2.4 \% ; 95 \%$ CI 2.2 to 2.7$)$. To note, positivity rates were at the same level in both genders at ages 60 and 66 years and did not increase by age (data not shown).
In total, 37 CRCs and 116 AAs were detected in the CRC screening pilot during the first year (table 2). The overall PPV for CRC per 100 positive FIT tests was 6.5 (4.6 to 8.9) and per 100 colonoscopies 8.9 (6.3 to 12.0). Both PPVs for CRC were only slightly higher in men than in women.

The overall PPV for AA per 100 positive FIT tests was 20.4 and was higher in men $(25.7 ; 95 \%$ CI 20.6 to 31.4$)$ than in women (15.5; 95\% CI 11.6 to 20.2) (table 2). The corresponding overall PPV for AA per 100 colonoscopies was 27.8 and accordingly higher in men (34.8; $95 \%$ CI 28.3 to 41.8$)$ than in women $(21.3 ; 95 \%$ CI 16.0 to 27.4$)$.

The overall CRC detection rate was 1.7 per 1000 participants with an assessable FIT (table 2). In men it was 1.8 (1.1 to 2.9 ) and in women 1.6 (0.9 to 2.4$)$. The corresponding AA detection rates were 5.3 in total and $7.2(5.6$ to 9.1 ) in men and 3.8 (2.8 to 5.0 ) in women.

There were no statistically significant differences between genders.

Table 2 Positivity rate, PPV and detection rate (n and \% with 95\% $\mathrm{Cl}$ ) for CRC and AA

\begin{tabular}{|c|c|c|c|c|c|c|}
\hline & \multicolumn{2}{|c|}{ Men } & \multicolumn{2}{|c|}{ Women } & \multicolumn{2}{|l|}{ All } \\
\hline & $\mathbf{n}$ & $\%(95 \% \mathrm{Cl})$ & $\mathbf{n}$ & $\%(95 \% \mathrm{Cl})$ & $\mathbf{n}$ & $\%(95 \% \mathrm{Cl})$ \\
\hline Positivity* & 272 & 2.8 (2.5 to 3.1$)$ & 296 & 2.4 (2.2 to 2.7$)$ & 568 & 2.6 (2.4 to 2.8$)$ \\
\hline PPV test for AA & 70 & 25.7 (20.6 to 31.4$)$ & 46 & 15.5 (11.6 to 20.2$)$ & 116 & $20.4(17.2$ to 24.0$)$ \\
\hline PPV colonoscopy for $\mathrm{CRC} \S$ & 18 & 9.0 (5.4 to 13.8$)$ & 19 & 8.8 (5.4 to 13.4$)$ & 37 & 8.9 (6.3 to 12.0$)$ \\
\hline CRC detection rate ${ }^{\star *}$ & 18 & 1.8 (1.1 to 2.9$)$ & 19 & 1.6 (0.9 to 2.4$)$ & 37 & 1.7 (1.1 to 2.3$)$ \\
\hline AA detection rate†† & 70 & $7.2(5.6$ to 9.1$)$ & 46 & 3.8 (2.8 to 5.0$)$ & 116 & 5.3 (4.4 to 6.3$)$ \\
\hline
\end{tabular}

${ }^{*}$ The proportion of participating persons with a test result at or above the cut-off level out of those with an assessable stool sample.

†The proportion of persons with CRC out of those with a positive test result.

$\ddagger$ The proportion of persons with AA out of those with a positive test result.

$\S$ The proportion of persons with CRC out of those who underwent a colonoscopy.

IThe proportion of persons with AA out of those who underwent a colonoscopy.

${ }^{\star *}$ The proportion of persons with CRC out of those with an assessable stool sample.

††The proportion of persons with AA out of those with an assessable stool sample.

AA, advanced adenoma; CRC, colorectal cancer; PPV, positive predictive value. 


\section{DISCUSSION}

CRC screening with FIT test has been running in Finland since April 2019 in nine municipalities. The design and strategy of the forthcoming national screening programme in 2022 will be based on the experiences and outcomes of this ongoing pilot. The Finnish CRC programme aims to narrow the gap between genders and thus has introduced different cut-off values of FIT for women and men. The first-year results reveal excellent participation and well-performing test and referral protocols. The gender-specific positivity rates were, however, lower than expected, especially in women. The PPVs and the detection rates of AAs were higher in men than in women. Nonetheless, gender-specific PPVs and detection rates of CRC were almost equal.

\section{Strengths and limitations of the study}

To our knowledge, this study is the first to report the performance of FIT screening with different screening schemes for men and women. The piloting phase enables us to establish the best possible FIT cut-offs for both genders before the implementation of the national programme. All performance indicators are reported separately for men and women and discussed accordingly. We have established an IT system enabling a real-time follow-up of each invitee throughout the screening chain. Additionally, we were able to link all invitees with the nationwide cancer and population data to verify cancer diagnoses and deaths.

Nine Finnish municipalities participated in the FIT pilot. The population of individuals aged 60-74years old in these municipalities accounted for approximately 9.5\% of all individuals of that age in Finland in 2014$2018 .^{26}$ Since $9.1 \%$ of all CRCs at ages $60-74$ years were diagnosed in their area in 2014-2018, ${ }^{14}$ we assume that the screening invitees represent well the CRC risk profile of the Finnish population aged 60-74years old. The small discrepancy between the percentages may reflect the slightly younger age structure of participating municipalities, where people aged 60-74 accounted for $16 \%$ of the total population. Elsewhere in Finland, the corresponding proportion is $20 \%{ }^{26}$

After a careful check-up we noticed that $6.3 \%$ of the current invitees had already been invited to the previous gFOBT screening. With a participation rate of $66 \%$ in the gFOBT programme in $2014,{ }^{27}$ approximately $4.4 \%$ of them may have participated in the CRC screening the second time in 2019. However, this hardly affects the level of performance indicators of the current study.

\section{Comparison with other studies}

After a 1-year follow-up, the positivity rates in the Finnish pilot were $2.8 \%$ for men and $2.4 \%$ for women. These rates were surprisingly close to each other, but lower than expected, especially in women. When comparing these with the published first-round positivity rates of a Finnish gFOBT programme in 2004-2007 (men 3.1\%, women $1.4 \%),{ }^{28}$ the current rates were lower in men and higher in women. Accordingly, the CRC detection rate was lower in men (1.8 vs 2.1 per 1000 participants) and higher in women (1.6 vs 1.0 per 1000 participants), but overall higher than in 2004-2007 (1.7 vs 1.5 per 1000 participants). The PPVs for CRC were similar. The number of AAs in the Finnish gFOBT programme had not been reported. Thus, we could only compare the detection rates and PPVs of all adenomas. These were higher in the FIT pilot compared with the gFOBT programme (12.3 vs 12.0 per 1000 participants for men, 8.4 vs 3.5 per 1000 participants for women). Accordingly, PPVs of all adenomas were also higher (44.1 vs 38.1 per 100 positives for men, 34.8 vs 21.0 per 100 positives for women). All in all, the first-year results of the FIT programme showed similar or slightly improved performance of CRC screening in men and clearly improved performance in women compared with the previous results. ${ }^{28}$

Where do our results lie in a wider European perspective? Table 3 compares the Finnish estimates with available first-round performance indicators of FIT screening at ages 60-69 years from Denmark, Italy, the Netherlands, Slovenia and Spain. ${ }^{18} 2029$ To note, the FIT tests and also their cut-offs differ by country, being $20 \mu \mathrm{g} \mathrm{Hg} / \mathrm{g}$ faeces in Italy, Slovenia, Spain and Denmark, and 15 and $47 \mu \mathrm{g}$ $\mathrm{Hg} / \mathrm{g}$ faeces in the Netherlands. Also, the age-standardised (world) CRC incidence rates vary, from 13.5 (Finland) to 29.3 (Slovenia) in men and from 11.3 (Finland) to 19.1 (Denmark) in women. ${ }^{30}$ Finally, the screening protocols also vary, and in many countries the invitees are only a subgroup of the target population due to exclusion of those with personal history of CRC, previous colonoscopy within 3-5 years or enrolment in a CRC surveillance programme. ${ }^{20}{ }^{31-33}$ In Spain, for example, these exclusions have reduced the number of target population for more than 20\%, from 583000 to $459000{ }^{33}$ In Slovenia, the target population is asked whether they agree to be invited. The low response rate to this query has reduced the amount of target population by more than $40 \% .{ }^{19}$

Table 3 shows that participation rates in the European FIT programmes vary from $57 \%$ to $79 \%$ and have been highest in Finland. This may at least partly be due to two reminder rounds, which seemed to improve the overall participation from $54 \%$ to $79 \%$ (table 1). The Finnish positivity rates were, however, the lowest of all countries. Considering the low incidence rates and the relatively high cut-off level in men, this is no surprise, but requires further action. Also, the detection rates of CRC, AA and any adenoma in Finland were the lowest. This is probably related to the low CRC incidence, but may also be due to chosen positivity protocol and the fact that all target populations in Finland are invited regardless of previous CRC or colonoscopy history. However, the Finnish PPVs of FIT for CRC as well as the PPVs of colonoscopy for CRC, AA and adenoma were comparable with those in other European countries. Even so, the PPVs of FIT for AA and adenoma were clearly lower in Finland than in other countries excluding Italy. Overall, the variability in performance indicators in the European FIT programmes 


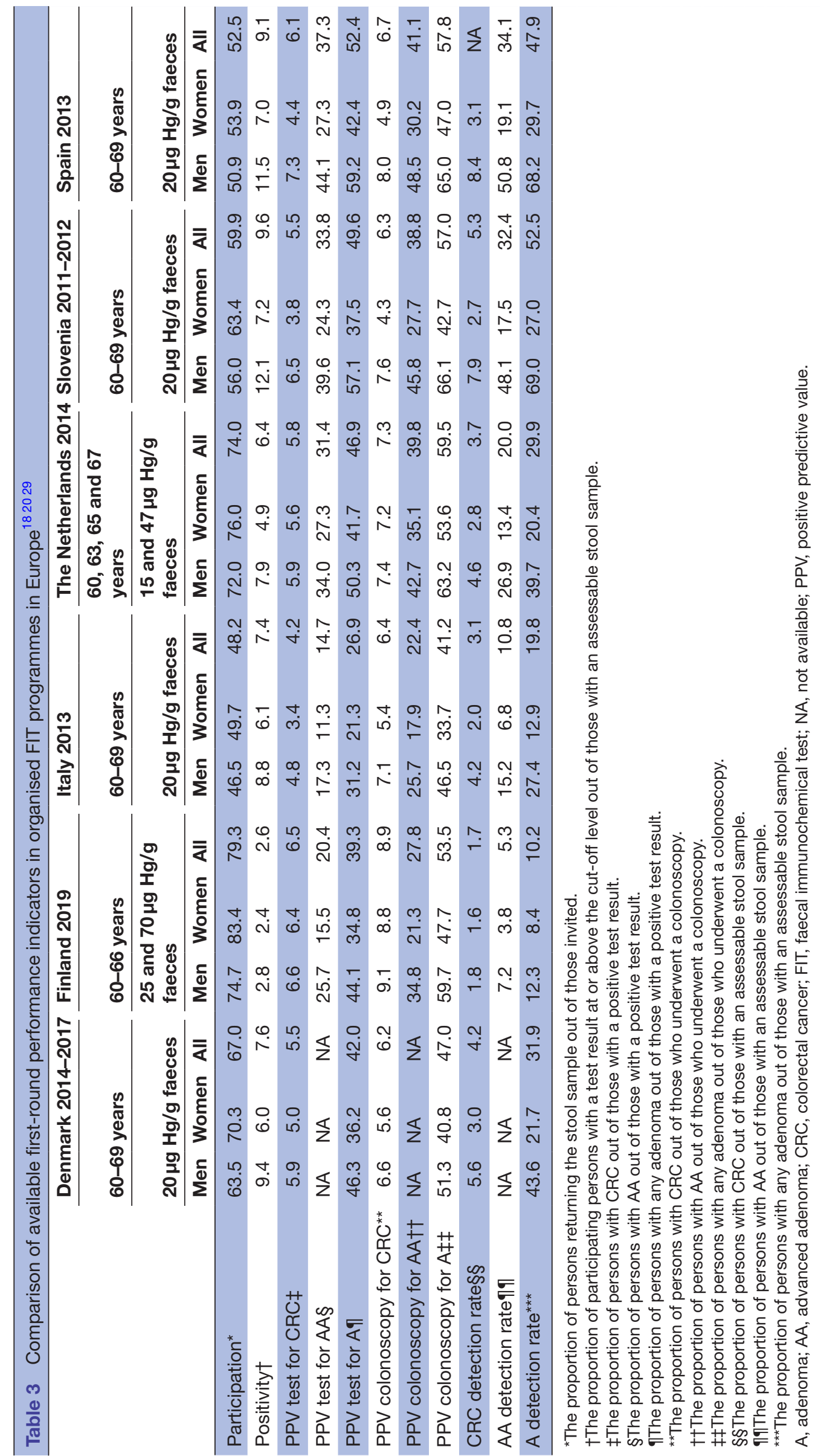


was wide. The figures from the Netherlands were most impressive and, according to the national report, have also persisted later. ${ }^{34}$

\section{CONCLUSION AND IMPLICATIONS}

The chosen FIT strategy in Finland has improved the performance of CRC screening slightly among men and clearly among women compared with the previous gFOBT screening and thus has narrowed the gap in the diagnostic performance between genders. The screening process functions well, the participation rates are excellent, and most PPVs are comparable with those of other European programmes. The positivity rates have, however, remained low, and the detection rates of CRC and AA are yet moderate, especially in men. In the future, along with the gradually expanding target age, we expect the positivity and detection rates to improve. Additionally, we have reduced the FIT cut-off level to $50 \mu \mathrm{g} / \mathrm{g}$ for men invited to screening in 2020. Also a modelling study has been launched to find the best possible FIT thresholds and target ages for the full national screening programme due in 2022.

\section{Author affiliations}

${ }^{1}$ Finnish Cancer Registry, Cancer Society of Finland, Helsinki, Finland

${ }^{2}$ Clinic of Gastroenterology, Helsinki University Central Hospital, Helsinki, Finland

${ }^{3}$ Finnish Cancer Registry, Cancer Society of Finland Institute for Statistical and

Epidemiological Cancer Research, Helsinki, Finland

${ }^{4}$ Department of Gastroenterology, Tampere University Hospital, Tampere, Finland

${ }^{5}$ Central Finland Health Care District, Jyväskylä, Finland

${ }^{6}$ Department of Surgery, Medical Research Center, Oulu University Hospital, Oulu, Finland

${ }^{7}$ Turku University, Turku, Finland

\section{Twitter Maija Jäntti @MaijaJantti}

Contributors TS planned the design and wrote the manuscript and acts as guarantor. SH collaborated in the planning phase and did the analyses. ML and LP prepared the baseline data, and ML and SH reviewed and coded the data. MF, AA, MH, MK, TR, MV, SH, MJ and NM codesigned the CRC screening pilot and interpreted the results and provided comments on the manuscript. The corresponding author attests that all authors meet the authorship criteria and that no others meeting the criteria have been omitted.

Funding The authors have not declared a specific grant for this research from any funding agency in the public, commercial or not-for-profit sectors.

Competing interests None declared.

Patient and public involvement Patients and/or the public were not involved in the design, or conduct, or reporting, or dissemination plans of this research.

Patient consent for publication Not required.

Ethics approval The study is based on registry data and was approved by the National Institute for Health and Welfare (reference: THL/356/5.05.00/2019).

Provenance and peer review Not commissioned; externally peer reviewed.

Data availability statement All data relevant to the study are included in the article or uploaded as supplementary information. Aggregated data may be made available on request to accredited researcher who submits a proposal that is admitted by the research group.

Open access This is an open access article distributed in accordance with the Creative Commons Attribution Non Commercial (CC BY-NC 4.0) license, which permits others to distribute, remix, adapt, build upon this work non-commercially, and license their derivative works on different terms, provided the original work is properly cited, appropriate credit is given, any changes made indicated, and the use is non-commercial. See: http://creativecommons.org/licenses/by-nc/4.0/.
ORCID iDs

Tytti Sarkeala http://orcid.org/0000-0002-0294-8757

Maija Jäntti http://orcid.org/0000-0001-6826-7551

\section{REFERENCES}

1 Council recommendation of 2. December 2003 on cancer screening. Official J Europ Union 2003:34-8.

2 Brenner H, Tao S. Superior diagnostic performance of faecal immunochemical tests for haemoglobin in a head-to-head comparison with guaiac based faecal occult blood test among 2235 participants of screening colonoscopy. Eur J Cancer 2013:49:3049-54.

3 Young GP, Symonds EL, Allison JE, et al. Advances in fecal occult blood tests: the fit revolution. Dig Dis Sci 2015;60:609-22.

4 Malila N, Oivanen T, Malminiemi O, et al. Test, episode, and programme sensitivities of screening for colorectal cancer as a public health policy in Finland: experimental design. BMJ 2008;337:a2261.

5 Pitkäniemi J, Seppä K, Hakama M, et al. Effectiveness of screening for colorectal cancer with a faecal occult-blood test, in Finland. BMJ Open Gastroenterol 2015;2:e000034.

6 Brenner H, Haug U, Hundt S. Sex differences in performance of fecal occult blood testing. Am J Gastroenterol 2010;105:2457-64.

7 Stegeman I, de Wijkerslooth TR, Stoop EM, et al. Risk factors for false positive and for false negative test results in screening with fecal occult blood testing. Int J Cancer 2013;133:2408-14.

8 Shaukat A, Mongin SJ, Geisser MS, et al. Long-term mortality after screening for colorectal cancer. $N$ Engl J Med 2013;369:1106-14

9 Massat NJ, Moss SM, Halloran SP, et al. Screening and primary prevention of colorectal cancer: a review of sex-specific and sitespecific differences. J Med Screen 2013;20:125-48.

10 Lash RH, Genta RM, Schuler CM. Sessile serrated adenomas: prevalence of dysplasia and carcinoma in 2139 patients. J Clin Pathol 2010;63:681e686

11 Malila N, Anttila A, Hakama M. Colorectal cancer screening in Finland: details of the National screening programme implemented in autumn 2004. J Med Screen 2005;12:28-32.

12 Färkkilä N, Torvinen S, Sintonen H, et al. Costs of colorectal cancer in different states of the disease. Acta Oncol 2015;54:454-62.

13 Published materials on colorectal cancer screening in the Finnish cancer registry. Available: https://cancerregistry.fi/screening/ organising-cancer-screening/

14 National cancer incidence figures of the Finnish Cancer Registry. Available: https://cancerregistry.fi/statistics/

15 National life expectancy figures of the statistics Finland,. Available: http://tilastokeskus.fi/til/kuol/2019/kuol_2019_2020-04-24_kuv_002 en.html

16 Lauby-Secretan B, Vilahur N, Bianchini F, et al. The IARC perspective on colorectal cancer screening. N Engl J Med 2018;378:1734-40.

17 Public Health England. Bowel cancer screening: programme overview, 2015. Available: https://www.gov.uk/guidance/bowelcancer-screening-programme-overview

18 Ponti A, Anttila A, Ronco G. 2nd report on the implementation of the Council recommendation on cancer screening. IARC, European Commission: Lyon, 2017.

19 Novak-Mlakar D, Kofol Brick T, Skrjanek AL, Colorectal cancer screening in Slovenia. Slovenian national colorectal cancer screening programme, National Institute of public health, 2018. Available: http://www.dpor.si/eng/wp-content/uploads/2018/11/DPOR_ POROCILO2018_SVIT_ang_posredovano.pdf

20 Njor SH, Friis-Hansen L, Andersen B, et al. Three years of colorectal cancer screening in Denmark. Cancer Epidemiol 2018;57:39-44.

21 ISD Scotland. Scottish bowel screening programme, 2019. Available: https://www.isdscotland.org/Health-Topics/Cancer/Bowel-Screening/

22 Nordcan database of cancer statistics for the Nordic countries: Denmark, Finland, Iceland, Norway, Sweden, Faroe Islands and Greenland. Available: http://www-dep.iarc.fr/NORDCAN/english/ frame.asp

23 Nationale prævalens screeningsrunde. Dansk tarmkræftscreeningsdatabase. Årsrapport 2017. Available: https:// www.sundhed.dk/content/cms/45/61245_dts_\%C3\%A5rsrapport2017 final.pdf

24 Blom J, Löwbeer C, Elfström KM, et al. Gender-specific cut-offs in colorectal cancer screening with FIT: increased compliance and equal positivity rate. J Med Screen 2019;26:92-7. 
25 East JE, Atkin WS, Bateman AC, et al. British society of gastroenterology position statement on serrated polyps in the colon and rectum. Gut 2017;66:1181-96.

26 National figures on municipality population of the statistics Finland. Available: http://pxnet2.stat.fi/PXWeb/pxweb/fi/StatFin/StatFin vrm_vaerak/?rxid=0797186c-2333-4511-ac56-1a90cad01292

27 Statistics of gFOBT-colorectal cancer screening in Finland in 2014,. Available: http://stats.cancerregistry.fi/joukkistilastot/suolisto.html

28 Malila N, Palva T, Malminiemi O, et al. Coverage and performance of colorectal cancer screening with the faecal occult blood test in Finland. J Med Screen 2011;18:18-23.

29 Toes-Zoutendijk E, van Leerdam ME, Dekker E, et al. Realtime monitoring of results during first year of dutch colorectal cancerscreening program and optimization by altering fecal immunochemical test cut-off levels. Gastroenterol 2017;152:767-75.
30 Global cancer observatory 2018, 2018. Available: https://gco.iarc.fr/

31 Rossi PG, Vicentini M, Sacchettini C, et al. Impact of screening program on incidence of colorectal cancer: a cohort study in Italy. Am J Gastroenterol 2015;110:1359-66.

32 Arana-Arri E, Idigoras I, Uranga B, et al. Population-based colorectal cancer screening programmes using a faecal immunochemical test: should faecal haemoglobin cut-offs differ by age and sex? BMC Cancer 2017;17:577.

33 Tepeš B, Bracko M, Novak Mlakar D, et al. Results of the FIT-based national colorectal cancer screening program in Slovenia. J Clin Gastroenterol 2017;51:e52-9.

34 Colorectal Cancer Screening Programme. Monitor 2017. Rotterdam: Netherlands Cancer Institute, 2017. https://www.rivm. $\mathrm{nl} /$ documenten/monitoring-and-evaluation-of-colorectal-cancerscreening-2017 